\title{
COMPARAÇÃO ENTRE LEITURAS DE RESISTÓGRAFO E IMAGENS TOMOGRÁFICAS NA AVALIAÇÃO INTERNA DE TRONCOS DE ÁRVORES
}

\author{
Francisco Martins de Almeida Rollo ${ }^{1}$, Mauro Angelo Soave Junior ${ }^{2}$, Sabrina Mieko Viana ${ }^{3}$, \\ Luciana Cavalcante Pereira Rollo ${ }^{4}$, Hilton Thadeu Zarate do Couto ${ }^{5}$, Demóstenes Ferreira da Silva Filho ${ }^{6}$
}

(recebido: 9 de fevereiro de 2011; aceito: 25 de janeiro de 2013)

RESUMO: A avaliação do risco de queda de árvores urbanas é uma tarefa complexa que demanda, sempre que possível, o auxílio de critérios e métodos confiáveis que possam fornecer informações mais precisas sobre o estado dos indivíduos avaliados. Dentre os instrumentos hoje utilizados, destaca-se o resistógrafo que permite a detecção de problemas biomecânicos das árvores, por meio de leituras de resistência à penetração de uma agulha. Recentemente, surgiu a técnica da tomografia de impulso para a detecção de podridão interna que se baseia no cálculo da velocidade de propagação das ondas mecânicas no interior do lenho, originando uma imagem tomográfica que permite observar áreas de maior e menor densidade correlacionadas à existência de lesões e processos de apodrecimento. A comparação desses dois métodos de avaliação de risco de queda foi feita com o intuito de avaliar a qualidade dos dados fornecidos pelo tomógrafo de impulso, analisando sua relação com os dados do resistógrafo. Obteve-se um modelo com alto coeficiente de determinação $\left(\mathrm{R}^{2}\right)$ de 0,9977 , mostrando que os dados fornecidos pelo tomógrafo possuem alta qualidade.

Palavras-chave: Risco de queda, resistógrafo, tomografia de impulso.

\section{COMPARISON BETWEEN RESISTOGRAPHY READINGS AND TOMOGRAPHIC IMAGES FOR INTERNAL ASSESSMENT IN TREES TRUNKS}

\begin{abstract}
Risk assessment of tree falls is essential for urban management and demands reliable methods and criteria capable of providing accurate information on assessed individuals. Among the tools currently used, resistograph stands out since it enables the detection of biomechanical problems through readings of needle penetration resistance. Recently, impulse tomography has been used as advanced technology to detect inner rottenness based on measurements of energy propagation velocity in inner tissues, generating a tomographic image that highlights higher and lower density areas related to lesions and rot processes. We compared these two risk assessment methods of trees to evaluate data reliability generated from impulse tomography, contrasting its results with data from resistograph. We obtained a model with high determination coefficient $\left(R^{2}\right)$ of 0.9977 , showing that data provided by impulse tomography are high quality and reliable.
\end{abstract}

Key words: Fall risks, resistograph, impulse tomography.

\section{INTRODUÇÃO}

A impermeabilização e a compactação do solo, a elevação das temperaturas decorrentes do fenômeno das ilhas de calor, as alterações na pluviosidade, a alta concentração de poluentes, a competição por espaço com os equipamentos urbanos (garagens, rede elétrica, dentre outros), assim como o vandalismo, diminuem a expectativa de vida das árvores nas cidades
(QUIGLEY, 2004). A baixa biodiversidade, ocasionada pelo plantio de poucas espécies também é um fator preocupante, uma vez que torna a floresta urbana mais suscetível a doenças e pragas. Esses elementos são os principais responsáveis pelo aumento do risco de queda de árvores em cidades.

Métodos que visam a averiguar o risco de queda dessas árvores auxiliam o manejo e ampliam a compreensão da dinâmica da floresta urbana, além de

${ }^{1}$ Gestor ambiental, Mestre em Recursos Florestais - Universidade de São Paulo/USP - Escola Superior de Agricultura Luiz de Queiroz/ESALQ - Av. Pádua Dias, 11 - Cx. P. 9 - 13418-900 - Piracicaba, SP, Brasil - francisco.rollo@yahoo.com.br

${ }^{2}$ Gestor ambiental, Mestrando em Recursos Florestais - Universidade de São Paulo/USP - Escola Superior de Agricultura Luiz de Queiroz/ESALQ Av. Pádua Dias, 11 - Cx. P. 9 - 13418-900 - Piracicaba, SP, Brasil - mauro.soave@usp.br

${ }^{3}$ Bióloga, Doutoranda em Recursos Florestais - Universidade de São Paulo/USP - Escola Superior de Agricultura Luiz de Queiroz/ESALQ - Av. Pádua Dias, 11 - Cx. P. 9 - 13418-900 - Piracicaba, SP, Brasil - smieko@usp.br

${ }^{4}$ Engenheira agrônoma, Doutoranda em Recursos Florestais - Universidade de São Paulo/USP - Escola Superior de Agricultura Luiz de Queiroz/ ESALQ - Av. Pádua Dias, 11 - Cx. P. 9 - 13418-900 - Piracicaba, SP, Brasil - lrollo@ usp.br

${ }^{5}$ Engenheiro agrônomo, Professor Doutor em Biometria Florestal - Universidade de São Paulo/USP - Escola Superior de Agricultura Luiz de Queiroz/ESALQ - Departamento de Ciências Florestais - Av. Pádua Dias, 11 - Cx. P. 9 - 13418-900 - Piracicaba, SP, Brasil - htzcouto@usp.br ${ }^{6}$ Engenheiro agrônomo, Professor Doutor em Agronomia (Produção Vegetal) - Universidade de São Paulo/USP - Escola Superior de Agricultura Luiz de Queiroz/ESALQ - Departamento de Ciências Florestais - Av. Pádua Dias, 11 - Cx. P. 9 -13418-900 - Piracicaba, SP, Brasil - dfilho@usp.br

Cerne, Lavras, v. 19, n. 2, p. 331-337, abr./jun. 2013 
prestar-se para a prevenção de acidentes decorrentes de queda de árvores.

Métodos de avaliação visual de indivíduos arbóreos auxiliam na identificação de problemas biomecânicos e fitossanitários, bem como na interpretação do risco de queda (ALBERS et al., 2003; MATTHECK, 1991; MATTHECK; BRELOER, 1997; MATTHECK; KUBLER, 1995; NIKLAS, 2002). No entanto, somente este tipo de avaliação pode não fornecer informações suficientes sobre o estado da árvore, pois depende de critérios subjetivos e é baseada, principalmente, nos aspectos externos de sua condição.

Pereira et al. (2007) destacam que a literatura especializada menciona diversas técnicas de análise não destrutivas do lenho das árvores que, de acordo com Nicolotti et al. (2003), seriam mais apropriadamente chamadas de "quase não destrutivas", já que em muitos casos, sondas, eletrodos e transdutores são inseridos dentro da árvore. Essas técnicas fornecem informações de pequenas porções da madeira, tornando-se, muitas vezes, necessário realizar medições em diferentes pontos do lenho para conhecer a seção investigada.

Costello e Quarles (1999) estudaram a capacidade de detecção de apodrecimento de cerne com a utilização de furadeira portátil e resistógrafo em árvores das espécies Eucalyptus globulus e Ulmus glabra. No caso da furadeira, constatou-se que a experiência do operador fez a diferença na detecção de apodrecimento e que apodrecimentos em estágios iniciais não são detectados por esse método. Com o resistógrafo, a experiência do operador foi indiferente, entretanto o equipamento superestimou apodrecimentos em estágio inicial, mas mostrou grande capacidade de localizar aqueles em estágio médio e avançado. Os autores ressaltam que o conhecimento a respeito do padrão de resposta esperada para tecidos saudáveis e lesionados da espécie avaliada é essencial para a correta interpretação das leituras do resistógrafo.

Entre os métodos existentes para a avaliação de lesões no tronco, a tomografia caracteriza-se por ser uma técnica menos invasiva, capaz de fornecer informações a respeito de seções transversais inteiras dos indivíduos avaliados com uma única medição. Essa pode ser realizada por impulso, ondas de ultrassom, campo elétrico, ondas eletromagnéticas, raios $\gamma$ e raios X (NICOLOTTI et al., 2003).

A tomografia de impulso baseia-se no princípio da cronometragem de ondas mecânicas, cuja velocidade do impulso no interior da árvore está altamente correlacionada com a densidade, módulo de elasticidade e umidade do tecido, para reconstrução de seções das árvores, gerando um gráfico de velocidade das ondas mecânicas, também chamado de imagem tomográfica (RINNTECH, 2005). Porém, por tratar-se de uma técnica nova, existem poucos trabalhos realizados com tomografia de impulso, acarretando problemas para a interpretação das imagens e para a avaliação da confiabilidade dos dados obtidos (LAWDAY; HODGES, 2000; LONSDALE, 1999).

Trabalhos comparando tomografia com outros métodos de avaliação interna de árvores foram realizados por Nicolotti et al. (2003), comparando tomografia elétrica, por ultrassom e georradar. Martinis et al. (2004) utilizaram o resistógrafo e tomografia por ultrassom na deteç̧ão de podridão interna do tronco de árvores de Fagus sylvatica, Gilbert e Smiley (2004) testaram a capacidade de precisão do tomógrafo de impulso em indivíduos de Quercus alba e Caria spp. enquanto Wang e Allison (2008) compararam os dados do tomógrafo de impulso com avaliação visual e leituras de tempo de percurso do impulso. Pereira et al. (2007) compararam dados de tomografia de impulso com dados de densitometria de raio-x e observaram haver boa correlação entre eles.

Os trabalhos acima citados apontam que a tomografia como um método eficaz, preciso e que traz maiores informações do estado interno das árvores que os outros métodos, já que propicia uma visão da seção transversal completa dos indivíduos e possibilita a localização exata da área afetada por apodrecimento (GILBERT; SMILEY, 2004; MARTINIS et al., 2004; NICOLOTTI et al., 2003).

Nesse contexto, a comparação entre tecnologias que auxiliem na avaliação de risco de queda de árvores mostrase como importante forma de se conhecer o funcionamento e o desempenho de tecnologias mais recentes, por meio da comparação com instrumentos hoje utilizados nessa análise e cujo funcionamento é amplamente estudado. Frente a essa demanda, o presente estudo propôs-se a comparar dados obtidos pelo resistógrafo e pelo tomógrafo de impulso, de forma a determinar a relação entre eles para avaliar a confiabilidade dos dados gerados pelo tomógrafo.

\section{MATERIAL E MÉTODOS}

Para avaliar as similaridades nas informações obtidas pela tomografia de impulso e pelo resistógrafo foram utilizados dois toretes sadios de Eucalyptus saligna Sm., provenientes de plantio de 21 anos de idade da Estação Experimental de Itatinga, pertencente à ESALQ/USP. 
No primeiro torete, com um metro de altura e $31 \mathrm{~cm}$ de diâmetro, obtiveram-se dados em três alturas, sendo uma na região central, uma a $20 \mathrm{~cm}$ da face superior e outra a $20 \mathrm{~cm}$ da face inferior. Para a obtenção da imagem tomográfica, utilizou-se o tomógrafo de impulso Arbotom ${ }^{\circledR}$ com onze sensores posicionados de forma equidistante $(8,8 \mathrm{~cm}$ entre os sensores ). Cada sensor recebeu pancadas de martelo, para a produção de ondas mecânicas, até que os erros apresentados na tabela Delta\% do software do aparelho fossem inferiores a $10 \%$ (RINNTECH, 2005).

Em cada uma das seções utilizadas para a obtenção das imagens tomográficas, foram realizadas quatro medições com resistógrafo modelo Resistograph 3450
- S. As medições radiais foram realizadas em intervalos de $90^{\circ}$, sendo que a posição da primeira medição foi coincidente com a posição do primeiro sensor utilizado para a obtenção das imagens tomográficas (Figura 1). A profundidade de penetração da agulha foi de cerca de $15,5 \mathrm{~cm}$, até que sua ponta encontrasse o centro da seção transversal. Tal método foi adotado para que a correta localização da passagem da sonda do resistógrafo pudesse ser identificada na imagem tomográfica, permitindo correlacionar as variáveis resistência à penetração e velocidade de propagação de onda mecânica. Essas linhas de passagem foram identificadas com a utilização do software de geoprocessamento TNT Mips versão 7.2. e recortadas das imagens.

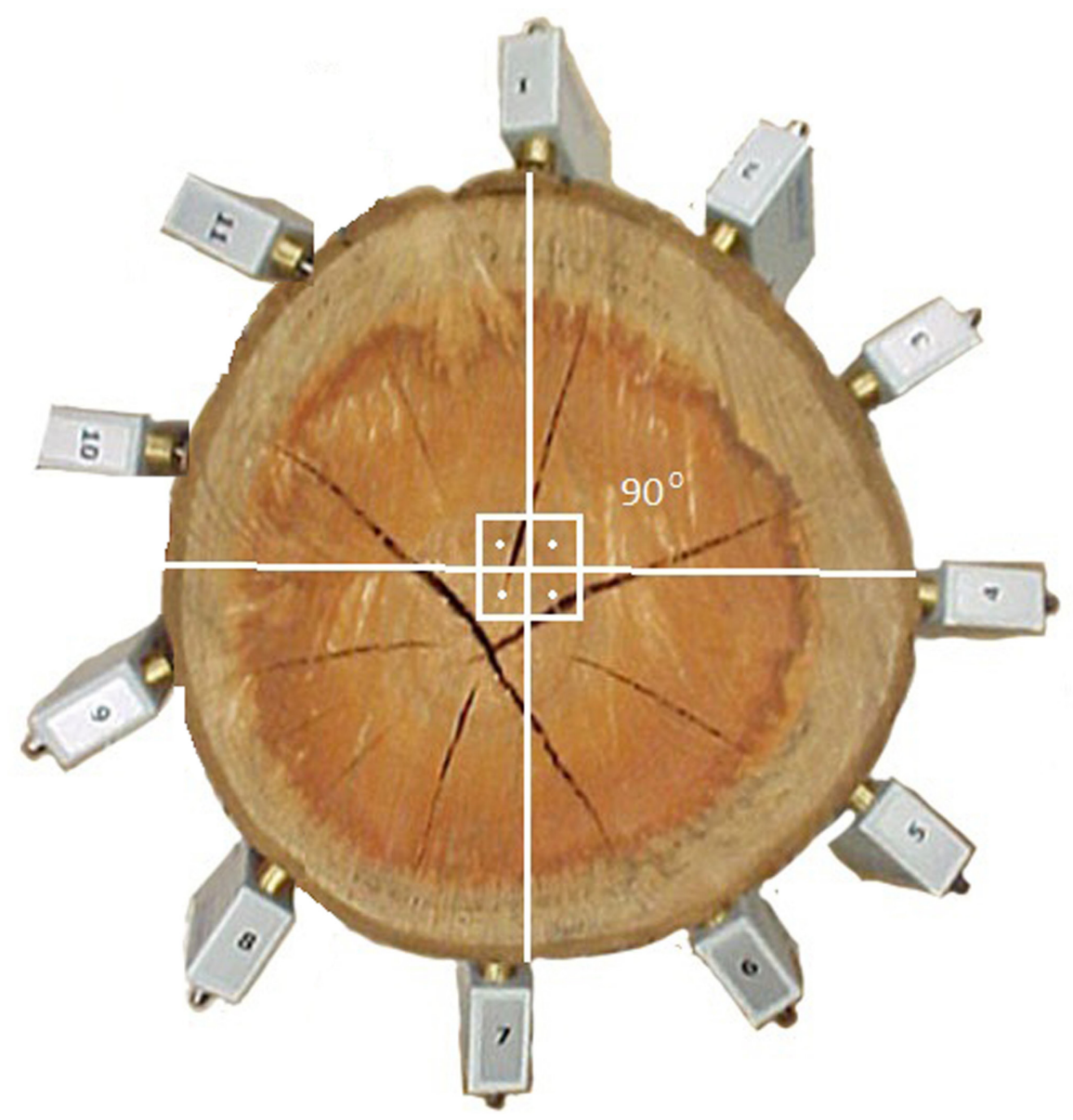

Figura 1 - Torete de Eucalyptus saligna com sensores do tomógrafo de impulso e seção transversal com marcação das posições de penetração da agulha do resistógrafo (a primeira coincidente com o primeiro sensor e as demais a cada $90^{\circ}$ ).

Figure 1 - Eucalyptus saligna log with impulse tomography sensors and cross-section with penetration position of resistography needle (the first coincided with first sensor and the others after $90^{\circ}$ ).

Cerne, Lavras, v. 19, n. 2, p. 331-337, abr./jun. 2013 
O mesmo processo para a obtenção de imagem tomográfica e leituras do resistógrafo foi realizado no segundo torete. Foi gerada uma única imagem tomográfica e quatro leituras de resistógrafo em seção transversal localizada a $20 \mathrm{~cm}$ da face superior da amostra de 1 metro de altura e 44,5 cm de diâmetro. Os sensores foram fixados em intervalos de $12,6 \mathrm{~cm}$ e a profundidade de penetração da agulha foi de cerca de $22,25 \mathrm{~cm}$.

Como os equipamentos utilizados apresentam suas leituras em escalas diferentes (Figura $2 \mathrm{~A}-\mathrm{B}$ ), fez-se necessária a padronização das escalas para que os dados pudessem ser comparados. Para o resistógrafo, foram calculados valores médios de resistência à penetração em intervalos de $3 \mathrm{~cm}$, sendo que cada média representava cerca de 3000 leituras, uma vez que o equipamento registra uma nova leitura para cada $0,01 \mathrm{~mm}$ perfurado. Para o tomógrafo de impulso, calculou-se a velocidade de propagação de onda mecânica em intervalos de $3 \mathrm{~cm}$, sendo que cada média representava cerca de 30 leituras, pois o equipamento fornece informações a cada $1 \mathrm{~mm}$.

Os Níveis de Cinza (NC) em cada uma das bandas ou canais (vermelho, verde e azul, representadas pelas letras $\mathrm{R} \mathrm{G} \mathrm{e} \mathrm{B,} \mathrm{respectivamente)} \mathrm{que} \mathrm{compõem} \mathrm{as} \mathrm{faixas} \mathrm{das}$ imagens tomográficas foram demarcados no software de geoprocessamento TNTMips em cada elemento da matriz da imagem (raster). Uma vez que a velocidade de propagação de onda mecânica está identificada pela composição de níveis de cinza em cada uma das bandas, foi construída uma palheta de cores e velocidades para cada uma das imagens, possibilitando a identificação da velocidade de propagação em cada elemento da matriz e a construção de um gráfico de velocidade para cada faixa avaliada.

Adotaram-se 155 classes de velocidade de propagação de onda mecânica por meio da formula (1), que possibilitou definir o intervalo de velocidade de cada uma das classes e os valores de níveis de cinza de cada uma das bandas enquadrados em cada classe:

$($ Vmax - Vmim)/155

Onde:

Vmax = velocidade máxima de propagação observada na imagem tomográfica;

Vmim = velocidade mínima observada na imagem tomográfica.

Com base nas leituras do resistógrafo e das velocidades obtidas pela tomografia de impulso, foram testados modelos linearizados, com seleção de variáveis feita pelo método de regressão linear passo a passo (Stepwise Linear), utilizando o programa SAS. Nos modelos, foram considerados os dados de resistência à penetração (variável dependente), velocidade de propagação da onda mecânica (variável independente) e o intercepto da equação (parâmetro $\hat{\beta}^{0}$ ) igual a zero, conforme apresentado na Tabela 1. Os valores dos coeficientes de determinação da regressão linear $\left(\mathrm{R}^{2}\right)$ obtidos e a simplicidade dos modelos foram considerados para a escolha do modelo adotado.
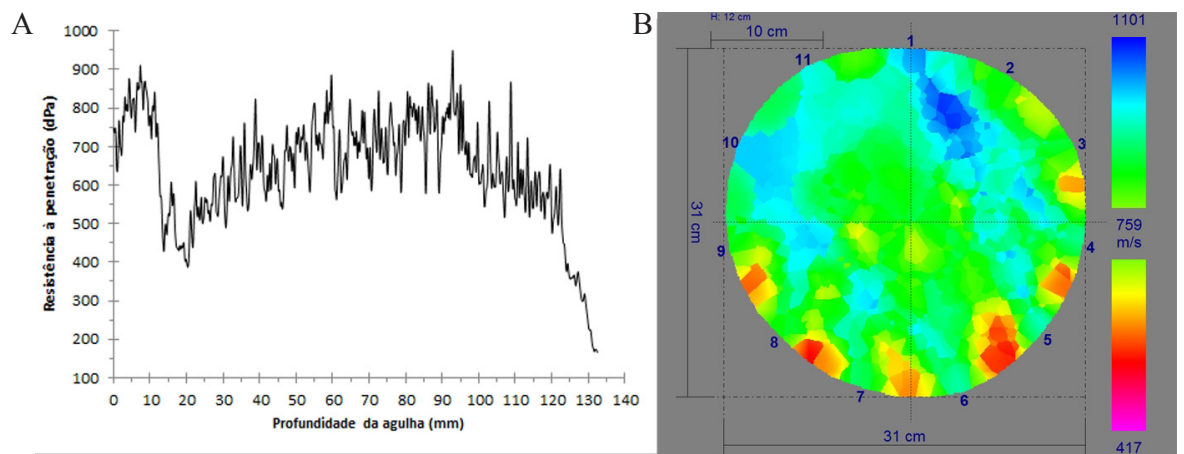

Figura 2 -A) Gráfico gerado pelo resistógrafo na análise interna do lenho de árvores, onde eixo x representa a distância percorrida pela agulha no tronco e o eixo y a resistência à penetração. B) Imagem obtida pelo tomógrafo de impulso de uma seção transversal, onde os tons de vermelho representam madeira menos densa, com menores velocidades de propagação de onda mecânica e os de azul madeira mais densa, com as maiores velocidades de propagação.

Figure 2-A) Graphic generated by resistograph inner tree wood analysis, where x axis represents the distance made by the needle on the trunk and $y$ axis the penetration resistance. B) Cross section image obtained by impulse tomograph, where red shades represents less dense wood, with low mechanical wave velocities and blue shades denser wood, with higher velocities.

Cerne, Lavras, v. 19, n. 2, p. 331-337, abr./jun. 2013 
Tabela 1 - Modelos da regressão linear passo a passo.

Table 1 - Stepwise models.

\begin{tabular}{cc}
\hline $\begin{array}{c}\text { Número do } \\
\text { Modelo }\end{array}$ & Fórmula correspondente \\
\hline 1 & $R E S I S=\hat{\beta} 1 *($ LTOMOG $)$ \\
2 & $R E S I S=\hat{\beta} 1 *(I T O M O G)+\hat{\beta} 2 *(L T O M O G)$ \\
3 & $R E S I S=e^{\hat{\beta} 1^{*}(L T O M O G)}$ \\
4 & $R E S I S=e^{\left(\hat{\beta} 1^{*}(\text { TOMOG })\right)+\left(\hat{\beta} 2^{*}(L T O M O G)\right)}$ \\
\hline
\end{tabular}

Nota: RESIS $=$ leitura do resistógrafo; $\mathrm{LTOMOG}=$ leitura do tomógrafo em escala logarítmica; ITOMOG = inverso da leitura do tomógrafo; TOMOG2 = leitura do tomógrafo elevada ao quadrado; $\hat{\beta}=$ parâmetro estimado; $e=$ exponencial.

\section{RESULTADOS E DISCUSSÃO}

A subdivisão das leituras do resistógrafo e das velocidades obtidas pela tomografia de impulso a cada três centímetros totalizaram 84 médias. Os parâmetros estimados e os coeficientes de determinação $\left(\mathrm{R}^{2}\right)$ para cada modelo testado estão dispostos na Tabela 2.

Embora o modelo 4 tenha apresentado um coeficiente de determinação $\left(\mathrm{R}^{2} 0,9978\right)$ superior dentre aqueles testados, este diferiu muito pouco do valor obtido pelo modelo 3. Dessa forma, optou-se pelo uso deste último $\left(\right.$ RESIS $\left.=e^{\left.\hat{\beta_{1}^{*}(L T O M O G)}\right)}\right)$, em função da sua maior simplicidade, já que este utiliza apenas uma vez a variável independente. Na Figura 3, são apresentados o diagrama de dispersão e linha de tendência do modelo 3 linearizado (LRESIS $=0,95855 *$ LTOMOG, onde: LRESIS é o logaritmo neperiano da resistência à penetração e LTOMOG é o logaritmo neperiano de velocidade de propagação da onda mecânica), bem como o gráfico de resíduos.

Com base no modelo adotado, podemos afirmar que os dados gerados pela aplicação do resistógrafo e os obtidos por meio da tomografia de impulso apresentam-

Tabela 2 - Parâmetros estimados e índice de qualidade de ajuste dos modelos testados.

Table 2 - Estimated parameters and tested models adjust quality index.

\begin{tabular}{cccc}
\hline $\mathrm{N}^{\circ}$ do modelo & $\hat{\beta} 1$ (erro padrão) & $\hat{\beta} 2$ (erro padrão) & Coeficiente de determinação $\left(\mathrm{R}^{2}\right)$ \\
\hline 1 & $101,95464_{(3,42883)}$ & --------------- & 0,9142 \\
2 & $-158129_{(78806)}$ & $129,42516_{(14,09858)}$ & 0,9182 \\
3 & $0,95855_{(0,005)}$ & ------------------ & 0,9977 \\
4 & $-2,32793 E-7_{(1,346701 E-7)}$ & $0,98627_{(0,01678)}$ & 0,9978 \\
\hline
\end{tabular}

Nota: $\hat{\beta} 1=$ primeiro parâmetro; $\hat{\beta} 2=$ segundo parâmetro.

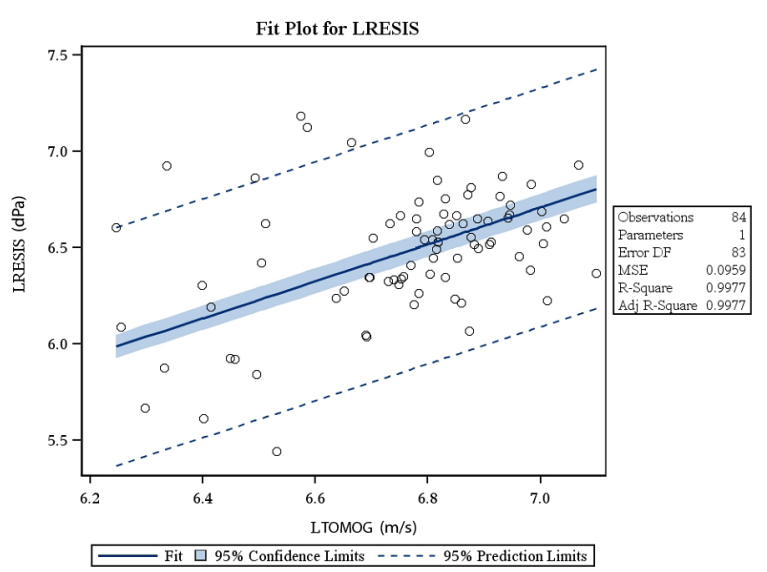

Figura 3 - Gráfico de dispersão incluindo modelo linearizado de resistência à penetração e velocidade de propagação de onda mecânica em 84 médias obtidas das amostras de toretes de Eucalipytus saligna Sm.

Figure 3 - Dispersion graphic and linearized model of penetration resistance and mechanic wave spread on 84 points obtained from samples of Eucalipytus saligna Sm.

Cerne, Lavras, v. 19, n. 2, p. 331-337, abr./jun. 2013 
se fortemente correlacionadas com o coeficiente de determinação $\left(\mathrm{R}^{2}\right)$ de 0,9977 . A resistência à penetração depende da densidade da madeira e a tomografia é sensível às suas variações no lenho.

Estudos conduzidos com clones de Eucalyptus sp. por Gouvêa et al. (2011) e Lima et al. (2007), indicaram que os resultados obtidos pelo resistógrafo apresentaram boa correlação com a densidade básica. Porém, ressalta-se que tanto neste estudo, quanto nos supracitados, foram realizados testes em amostras sem lesões. Costello e Quarles (1999), consideraram o resistógrafo como uma ferramenta capaz de identificar áreas lesionadas, assim pode-se sugerir que a tomografia de impulso também pode ser capaz de identificar tais áreas lesionadas, considerando a alta correlação obtida entre os dados de ambas as tecnologias em tecidos saudáveis. A vantagem da tomografia em relação ao resistógrafo se deve ao fato da primeira prover informações a respeito de toda uma seção transversal avaliada, enquanto o segundo permite leituras lineares, sendo necessárias diversas leituras para que a avaliação de uma seção transversal possa ser realizada acertadamente (NICOLOTTI et al., 2003). Outra vantagem da tomografia de impulso é a geração de uma imagem tomográfica que permite a localização das áreas lesionadas na seção avaliada.

\section{CONCLUSÕES}

As informações geradas pelas leituras de resistógrafo e pelas imagens tomográficas mostraram-se fortemente correlacionadas, de acordo com o modelo $R E S I S=e^{\hat{\beta} 1^{*}(L T O M O G)}\left(\mathrm{R}^{2}=0,9977\right)$. Portanto, os dados fornecidos pela tomografia de impulso são confiáveis.

Por gerar uma imagem da seção transversal inteira, a tomografia de impulso apresenta dados mais completos do que os gerados pelo resistógrafo (NICOLOTTI et al., 2003). No entanto, estudos com árvores vivas, com lesões internas e de diferentes espécies e densidades devem ser realizados para maior conhecimento dessa tecnologia.

\section{REFERÊNCIAS}

ALBERS, J. S.; POKORNY, J. D.; JOHNSON, G. R. How to detect and assess hazardous defects in trees. In: POKORNY, J. D. (Coord.). Urban tree risk management: a community guide to program design and implementation. Saint Paul: USDA Forest Service, 2003. p. 41-116. (Technical Paper, NA-TP-03-03).

Cerne, Lavras, v. 19, n. 2, p. 331-337, abr./jun. 2013
COSTELLO, L. R.; QUARLES, S. L. Detection of wood decay in blue gum and elm: an evaluation of the resistograph and the portable drill. Journal of Arboriculture, Champaign, v. 25, n. 6, p. 311-317, Nov. 1999.

GILBERT, E. A.; SMILEY, T. Picus Sonic tomography for the quantification of decay in white oak (Quercus alba) and hickory (Carya spp.). Journal of Arboriculture, Champaign, v. 30, n. 5, p. 277-281, Sept. 2004.

GOUVÊA, A. F. G.; TRUGILHO, P. F.; GOMIDE, J. L.; SILVA, J. R. M.; ANDRADE, C. R.; ALVES, I. C. N. Determinação da densidade básica das madeiras de Eucalyptus por diferentes métodos não destrutivos. Revista Árvore, Viçosa, v. 35, n. 2, p. 349-358, mar./abr. 2011.

LAWDAY, G.; HODGES, P. A. The analytical use of stress waves for the detection of decay in standing trees. Forestry, Oxford, v. 73, n. 5, p. 447-456, 2000.

LIMA, J. T.; SARTÓRIO, R. C.; TRUGILHO, P. F.; CRUZ, C. R.; VIEIRA, R. S. Uso do resistógrafo para estimar a densidade básica e a resistência à perfuração da madeira de Eucalyptus. Scientia Forestalis, Piracicaba, n. 75, p. 85-93, set. 2007.

LONSDALE, D. Principles of tree hazard assessment and management. Norwich: Her Majesty's Stationary Office, 1999. 388 p. (Forestry Commission Research for Amenity Trees, 7).

MARTINIS, R.; SOCCO, L. V.; SAMBUELLI, L.; NICOLOTTI, G.; SCHIMITT, O.; BUCUR, V. Tomographie ultrasonore pour les arbres sur pied. Annals of Forest Science, Champenoux, v. 61, n. 2, p. 157-162, 2004.

MATTHECK, C. Trees: the mechanical design. New York: Springer-Verlag, 1991. 121 p. (Springer Series in Wood Science).

MATTHECK, C.; BRELOER, R. C. The body language of trees: a handbook for failure analysis. London: The Stationery Office, 1997. $239 \mathrm{p}$.

MATTHECK, C.; KUBLER, H. Wood: the internal optimization of trees. New York: Springer-Verlag, 1995. 129 p. (Springer Series in Wood). 
NICOLOTTI, G.; SOCCO, L. V.; MARTINIS, R.; GODIO, A.; SAMBUELLI, L. Application and comparison of three tomographic tecniques for detection of decay in trees. Journal of Arboriculture, Champaign, v. 29, n. 2, p. 66-78, 2003.

NIKLAS, K. J. Wind, size and tree safety. Journal of Arboriculture, Champaign, v. 28, n. 2, p. 84-93, Mar. 2002.

PEREIRA, L. C.; SILVA FILHO, D. F.; TOMAZELO FILHO, M.; COUTO, H. T. Z.; MOREIRA, J. M. M. Á. P.; POLIZEL, J. L. Tomografia de impulso para avaliação do interior do lenho de árvores. Revista da Sociedade Brasileira de Arborização Urbana, Piracicaba, v. 2, n. 2, p. 65-75, 2007.
QUIGLEY, M. F. Street trees and rural conditions: will long-lived trees reach full size in urban conditions? Urban Ecosystems, New York, v. 7, p. 29-39, 2004.

RINNTECH. User manual: arbotom 3-D tree impulse tomograph. Version 1.59 for Microsoft Windows 98, 2000, XP. Heidelberg: Microsoft, 2005. 42 p.

WANG, X.; ALLISON, B. Decay detection in red oak trees using a combinations of visual inspection, acoustic testing, and resistance microdrilling. Arboriculture \& Urban Forestry, Champaign, v. 34, n. 1, p. 1-4, Jan. 2008.

Cerne, Lavras, v. 19, n. 2, p. 331-337, abr./jun. 2013 
